\title{
Real-world outcomes of anti-VEGF treatment for retinal vein occlusion in Portugal
}

\author{
Sara Vaz-Pereira, ${ }^{1,2}$, Inês P. Marques ${ }^{3}$, João Matias ${ }^{4}$, Filipe Mira ${ }^{5}$, Lígia Ribeiro ${ }^{6}$, Rita Flores ${ }^{7,8}$; on behalf of the Portuguese \\ Multicenter Study Group of Retinal Vascular Diseases (PORTUGAL)*
}

${ }^{1}$ Department of Ophthalmology, Hospital de Santa Maria, Centro Hospitalar Lisboa Norte, Lisbon - Portugal
${ }^{2}$ Department of Ophthalmology, Faculty of Medicine, Universidade de Lisboa, Lisbon - Portugal
${ }^{3}$ AIBILI Association for Innovation and Biomedical Research on Light and Image, Coimbra - Portugal
${ }^{4}$ Department of Ophthalmology, Centro Hospitalar do Baixo Vouga, Aveiro - Portugal
${ }^{5}$ Department of Ophthalmology, Centro Hospitalar Médio Tejo, EPE, Torres Novas - Portugal
${ }^{6}$ Department of Ophthalmology, Centro Hospitalar Vila Nova de Gaia e Espinho, Vila Nova de Gaia - Portugal
${ }^{7}$ Department of Ophthalmology, Centro Hospitalar de Lisboa Central, Lisbon - Portugal
${ }^{8}$ Department of Ophthalmology, Nova Medical School, Lisbon - Portugal

* Members of the Portuguese Mutlicenter Study Group of Retinal Vascular Diseases (PORTUGAL) are listed in the Appendix

\begin{abstract}
Purpose: Retinal vein occlusion (RVO) is an important cause of visual disability in the modern world. We aim to evaluate the real-world outcomes of patients with RVO treated with anti-vascular endothelial growth factor (VEGF) in Portugal.

Methods: We performed a retrospective, observational, multicenter study including 8 centers across Portugal and 200 patients treated with either ranibizumab or bevacizumab. Data were collected at 3 time points: time of diagnosis ( 0 time point) and 6 and 12 months after initiating treatment. Demographic and clinical data were collected. Results: Median visual acuity (VA) and central macular thickness (CMT) improved in the branch RVO (BRVO), central RVO (CRVO), bevacizumab, and ranibizumab groups at 6 and 12 months compared to baseline, with CMT improving further only in the CRVO and ranibizumab groups between 6 and 12 months $(p=0.002$ and $p=0.001$, respectively). The CMT was lower in the ranibizumab group compared to the bevacizumab group both at 6 and 12 months $(p<0.02)$. Median CMT improved in both the good and poor baseline VA groups at 6 and 12 months compared to baseline $(p<0.001)$. Median VA only improved for the group with poor baseline VA at 6 and 12 months of follow-up $(p<0.001)$. Regression analysis identified several baseline variables as predictors of visual outcomes at 6 and 12 months, with different results depending on the analyzed group.

Conclusions: Both treatments were effective, although less effective than results reported in clinical trials. The morphologic response was better with ranibizumab compared to bevacizumab, although functionally there were no differences.
\end{abstract}

Keywords: Anti-VEGF, Retinal vein occlusion, Visual prognosis

\section{Introduction}

Retinal vein occlusions (RVO) are the second most frequent retinal vasculopathy after diabetic retinopathy. Retinal vein occlusions have a global age- and sex-standardized prev-

Accepted: January 29, 2017

Published online: February 28, 2017

Corresponding author:

Rita Flores, MD

Department of Ophthalmology

Hospital de Santo António dos Capuchos

Centro Hospitalar de Lisboa Central

Alameda de Santo António dos Capuchos

1169-050 Lisbon, Portugal

ritamariaflores@gmail.com alence of 5.20 per $1,000: 4.42$ per 1,000 and 0.80 per 1,000 for branch RVO (BRVO) and central RVO (CRVO), respectively. It is estimated that 16.4 million adults are affected by RVO worldwide, corresponding to 13.9 million with BRVO and 2.5 million with CRVO (1).

The classification of RVO into BRVO and CRVO is based on the anatomical site of the vascular occlusion. Although the pathogenesis of RVO is not yet fully understood, an increased secretion of the vascular endothelial growth factor (VEGF) is observed, and therefore the use of anti-VEGF agents by intravitreal injections has become common (2).

Many treatment regimens have been suggested with anti-VEGF agents, such as monthly injections or injections pro re nata (PRN), but the ideal regimen has not been defined (2) and management in the long-term of RVO-related complications and visual loss has not been established (1). 
Among other therapeutic indications, ranibizumab and aflibercept are approved by the Food and Drug Administration and European Medicines Agency for the treatment of visual impairment due to macular edema secondary to BRVO or CRVO (2-4), while bevacizumab is used off-label worldwide $(2,5,6)$ if ranibizumab or aflibercept are unavailable or due to economic considerations (6).

It is of the utmost importance to understand how patients with RVO are being treated in a real-world setting, outside clinical trials, in order to identify measures that can be implemented to improve the visual outcomes of these patients.

The aim of this study was to characterize, in a real-world setting, patients with RVO treated with anti-VEGF in Portugal, and evaluate the respective functional and anatomical outcomes.

\section{Methods}

\section{Study design}

This retrospective, observational, multicenter study included 8 centers across Portugal.

\section{Participants and inclusion and exclusion criteria}

Files from patients with RVO were retrospectively reviewed between January and December 2015. Inclusion criteria were age 18 years or older, diagnosis of CRVO or BRVO with central macular edema, anti-VEGF as primary treatment (ranibizumab or bevacizumab), and follow-up of at least 12 months. Exclusion criteria included macular edema secondary to causes other than RVO (e.g., age-related macular degeneration, diabetic retinopathy, central serous retinopathy, uveitis), previous macular laser photocoagulation, previous anti-VEGF treatment due to conditions other than RVO, previous intravitreal or sub-Tenon corticosteroid injection, and history of vitreoretinal surgery.

This study was conducted according to the tenets of the Declaration of Helsinki. All participants provided written informed consent.

\section{Data collection}

Data were collected at 3 time points: at the time of diagnosis ( 0 time point) and 6 and 12 months after initiating treatment.

Clinical and demographic data collected included age, sex, type of RVO (CRVO or BRVO), comorbidities, best-corrected visual acuity (BCVA), central macular thickness (CMT), and phakic status. Treatment data included number of injections, type of anti-VEGF used, time to treatment, and adjuvant laser or corticosteroid therapy.

\section{Statistical analysis}

After assessing the normality of all variables using the Kolmogorov-Smirnov test, nonparametric statistics methods were used. For statistical purposes, Snellen fractions were converted to the logarithm of the minimum angle of reso- lution ( $\log M A R)$ by a standard conversion method (7). Data were analyzed considering all patients and 6 subgroups: CRVO or BRVO, ranibizumab or bevacizumab treatment, and good or poor baseline visual acuity, defined as logMAR $\leq 0.30$ and $\log M A R \geq 1.00$, respectively. Between-group analysis was performed using the Mann-Whitney $U$ test or the $\chi^{2}$ test, respectively, for continuous and discrete variables. Within-group analysis was performed using the Friedman or Wilcoxon test, as appropriate. Exploratory univariate analyses were performed, followed by multivariate regression analyses to determine predictors of BCVA and CMT at 6 and 12 months, depending on the 6 studied subgroups. The variables selected for the multivariate analyses were those found to be statistically significant from the univariate analyses as well as clinically relevant. The Bonferroni correction was applied to both univariate and multivariate analyses as needed. Tests were considered significant at $\alpha=0.05$ significance level (2-sided).

\section{Results}

A total of 200 eyes from 200 patients with central macular edema secondary to RVO were included in this study. There were 124 eyes with BRVO and 76 eyes with CRVO.

\section{Study population and baseline values}

Baseline demographics and clinical characteristics according to type of RVO and for the total population sample are shown in Table I. There were no significant differences between the studied variables when comparing patients with BRVO or CRVO, except for CMT, which was higher for patients with CRVO $(p<0.001)$. There were no differences between groups concerning baseline BCVA or age.

\section{Best-corrected visual acuity and CMT over the 12-month study period according to type of RVO}

Analysis of the 3 time points-baseline, 6 months, and 12 months-showed that the median BCVA improved in both the BRVO and CRVO groups at 6 and 12 months compared to baseline $(p<0.01)$, from 0.70 to 0.43 and 0.44 and from 0.80 to 0.70 and 0.70 , respectively. No differences were found between 6 and 12 months of follow-up. Between-group analysis showed that, at both 6 and 12 months, median BCVA was better in the BRVO group ( $p<0.005)$. Median CMT decreased in both the BRVO and CRVO groups at 6 and 12 months compared to baseline, but only the CRVO group showed a statistically significant decrease, also between 6 and 12 months ( $p<0.001$ and $p=0.002$, respectively). Between-group analysis revealed that, at both baseline and 6 months, median CMT was lower in the BRVO group $(p<0.005)$, with no differences at 12 months (Tab. II).

Best-corrected visual acuity and CMT over the 12-month study period according to treatment

Analysis of the 3 time points-baseline, 6 months, and 12 months-showed that the median BCVA improved in both the ranibizumab and bevacizumab groups at 6 and 12 months 
TABLE I - Baseline demographics and clinical characteristics according to type of retinal vein occlusion and for the total population sample

\begin{tabular}{lccr}
\hline Parameter & BRVo & CRVO & Total \\
\hline No. (\%) & $124(62.0)$ & $76(38.0)$ & $200(100.0)$ \\
Age, $y$, median (min-max) & $70.0(33.0-91.0)$ & $69.5(33.0-87.0)$ & $70.0(33.0-91.0)$ \\
Female, \% & 56.5 & 40.8 & 50.5 \\
Ranibizumab, $n$ (\%) & $65(52.4)$ & $42(55.3)$ & $107(53.3)$ \\
BCVA, logMAR, median (min-max) & $0.70(0.00-2.10)$ & $0.80(0.10-3.00)$ & $0.70(0.00-3.00)$ \\
CMT, $\mu$ m, median (min-max) & $535.0^{\text {a }}(291.0-981.0)$ & $693.5^{\text {a }}(292.0-1430.0)$ & $599.0(291.0-1430.0)$ \\
LogMAR $\leq 0.30, \%$ & 20.2 & 11.8 & 17.0 \\
LogMAR $\geq 1.00, \%$ & 37.9 & 42.1 & 39.5 \\
Cardiovascular comorbidities, \% ${ }^{\mathrm{b}}$ & 9.2 & 23.6 & 14.1 \\
Glaucoma & 4.9 & 4.1 & 1 \\
Pseudophakic, \% & 13.9 & 12.0 & 13.6 \\
\hline
\end{tabular}

${ }^{a} p<0.001$

b Hypertension + type 2 diabetes mellitus.

$\mathrm{BCVA}=$ best-corrected visual acuity; $\mathrm{BRVO}=$ branch retinal vein occlusion; $\mathrm{CMT}=$ central macular thickness; $\mathrm{CRVO}=$ central retinal vein occlusion; logMAR $\leq 0.30=$ good vision at baseline; $\log M A R \geq 1.00=$ poor vision at baseline.

TABLE II - Best-corrected visual acuity (BCVA) and central macular thickness (CMT) in patients with branch retinal vein occlusion (BRVO) and patients with central retinal vein occlusion (CRVO)

\begin{tabular}{lccc}
\hline Parameter & Baseline & $\mathbf{6}$ months & 12 months \\
\hline $\begin{array}{l}\text { BCVA, logMAR } \\
\text { BRVO }\end{array}$ & $0.70(0.60)$ & $0.43(0.45)^{\mathrm{b}}$ & $0.44(0.50)^{\mathrm{b}}$ \\
CRVO & $0.80(0.80)$ & $0.70(0.79)^{\mathrm{b}}$ & $0.70(0.90)^{\mathrm{b}}$ \\
CMT, $\mu m^{\mathrm{c}}$ & & & \\
BRVO & $535.0(257.0)^{\mathrm{b}}$ & $333.0(145.0)^{\mathrm{b}}$ & $309.0(148.0)$ \\
CRVO & $693.5(289.0)^{\mathrm{b}}$ & $404.0(378.0)^{\mathrm{b}, \mathrm{d}}$ & $303.0(221.0)^{\mathrm{d}}$ \\
\end{tabular}

${ }^{a} p<0.01$ Within groups, for differences between baseline and 6 months and baseline and 12 months.

${ }^{\mathrm{b}} \mathrm{p}<0.005$ Between groups.

c $p<0.001$ Within groups, for differences between baseline and 6 months and baseline and 12 months.

${ }^{\mathrm{d}} \mathrm{p}=0.002$.

All values presented as median (interquartile range).

compared to baseline $(p<0.001)$, from 0.70 to 0.50 and 0.50 and from 0.80 to 0.60 and 0.55 , respectively, with no difference between 6 and 12 months. There were no significant differences between groups in the BCVA parameter at both 6 and 12 months. Median CMT decreased in both the ranibizumab and bevacizumab groups at 6 and 12 months compared to baseline, but only the ranibizumab group showed a statistically significant decrease, also between 6 and 12 months $(p<0.001$ and $p<0.001$, respectively). Between-group analysis revealed that, at both 6 and 12 months, median CMT was lower in the ranibizumab group $(p<0.02)$ (Tab. III). Also, more patients in the bevacizumab group underwent rescue grid laser photocoagulation during follow-up (66.3\% vs $29.9 \%$, p<0.001).
TABLE III - Best-corrected visual acuity (BCVA) and central macular thickness (CMT) over the 12-month study period according to treatment

\begin{tabular}{lccc}
\hline Parameter & Baseline & $\mathbf{6}$ months & 12 months \\
\hline Ranibizumab & & & \\
BCVA, logMAR & 0.70 & $0.50(-0.10$ & 0.50 \\
& $(0.00-1.70)$ & to 1.68$)$ & $(0.00-1.66)$ \\
CMT, $\mu \mathrm{m}$ & 555.0 & $329.0^{c}$ & $281.0^{c}$ \\
& $(291.0-1,430.0)$ & $(129.0-1,161.0)$ & $(143.0-1,489.0)$ \\
Bevacizumab & & & \\
BCVA, logMAR & 0.80 & 0.60 & 0.55 \\
& $(0.10-3.00)$ & $(0.00-3.00)$ & $(0.00-3.00)$ \\
CMT, $\mu m$ & 611.0 & $361.0^{c}$ & $341.5^{c}$ \\
& $(292.0-1,306.0)$ & $(194.0-1,130.0)$ & $(164.0-1,308.0)$ \\
\hline
\end{tabular}

a $p<0.001$ Within groups, for differences between baseline and 6 months and baseline and 12 months for both BCVA and CMT.

${ }^{b} \mathrm{p}=0.001$ Within group, for CMT difference between 6 and 12 months.

${ }^{c} p<0.02$ Between groups.

All values presented as median (minimum-maximum).

\section{Best-corrected visual acuity and CMT over the 12-month study period according to BCVA at baseline}

Median CMT improved in both groups at 6 and 12 months compared to baseline $(p<0.001)$, with a further decrease between 6 and 12 months for the group with poor vision at baseline $(p=0.012)$. Median BCVA only improved in the poor vision group at 6 and 12 months compared to baseline $(p<0.001)$, with no difference between 6 and 12 months, from 1.30 to 0.90 and 0.89 , respectively (Tab. IV). Concerning baseline characteristics, the group with good visual acuity at baseline was younger compared to the group with poor visual acuity, with a median (minimum-maximum) age of 63.0 (33.0- 
TABLE IV - Best-corrected visual acuity (BCVA) and central macular thickness (CMT) over the 12-month study period according to BCVA at baseline

\begin{tabular}{lccc}
\hline Parameter & Baseline & $\mathbf{6}$ months & $\mathbf{1 2}$ months \\
\hline LogMAR $\leq 0.30$ & & & $0.14^{\mathrm{a}}(0.00-1.50)$ \\
BCVA, logMAR & $0.30^{\mathrm{a}}(0.00-0.30)$ & $0.20^{\mathrm{a}}(-0.10$ to 0.70$)$ & $321.5(210.0-798.0)$ \\
CMT, $\mu m^{\mathrm{b}}$ & $449.5^{\mathrm{a}}(306.0-795.0)$ & $346.0(197.0-644.0)$ & \\
LogMAR $\geq 1.00$ & & & $0.89^{\mathrm{a}}(0.14-3.00)$ \\
BCVA, logMAR & $1.30^{\mathrm{a}}(1.00-3.00)$ & $326.0(129.0-1,161.0)$ & $282.5(143.0-1,489.0)$ \\
CMT, $\mu m^{\mathrm{c}}$ & $700.0^{\mathrm{a}}(325.0-1,430.0)$ & $(0.10-3.00)$ & \\
\hline
\end{tabular}

a $p<0.001$ Between groups.

${ }^{\mathrm{b}} \mathrm{p}<0.001$ Within group, for differences between baseline and 6 months and baseline and 12 months.

${ }^{c} p<0.001$ Within group, for difference between baseline and 6 and 12 months, with $p=0.012$ for difference between 6 and 12 months.

$\log M A R \leq 0.30=$ good vision at baseline; $\log M A R \geq 1.00=$ poor vision at baseline.

All values presented as median (minimum-maximum).

TABLE V - Predictors of best-corrected visual acuity (BCVA) and central macular thickness at 6 and 12 months, depending on the 6 studied subgroups

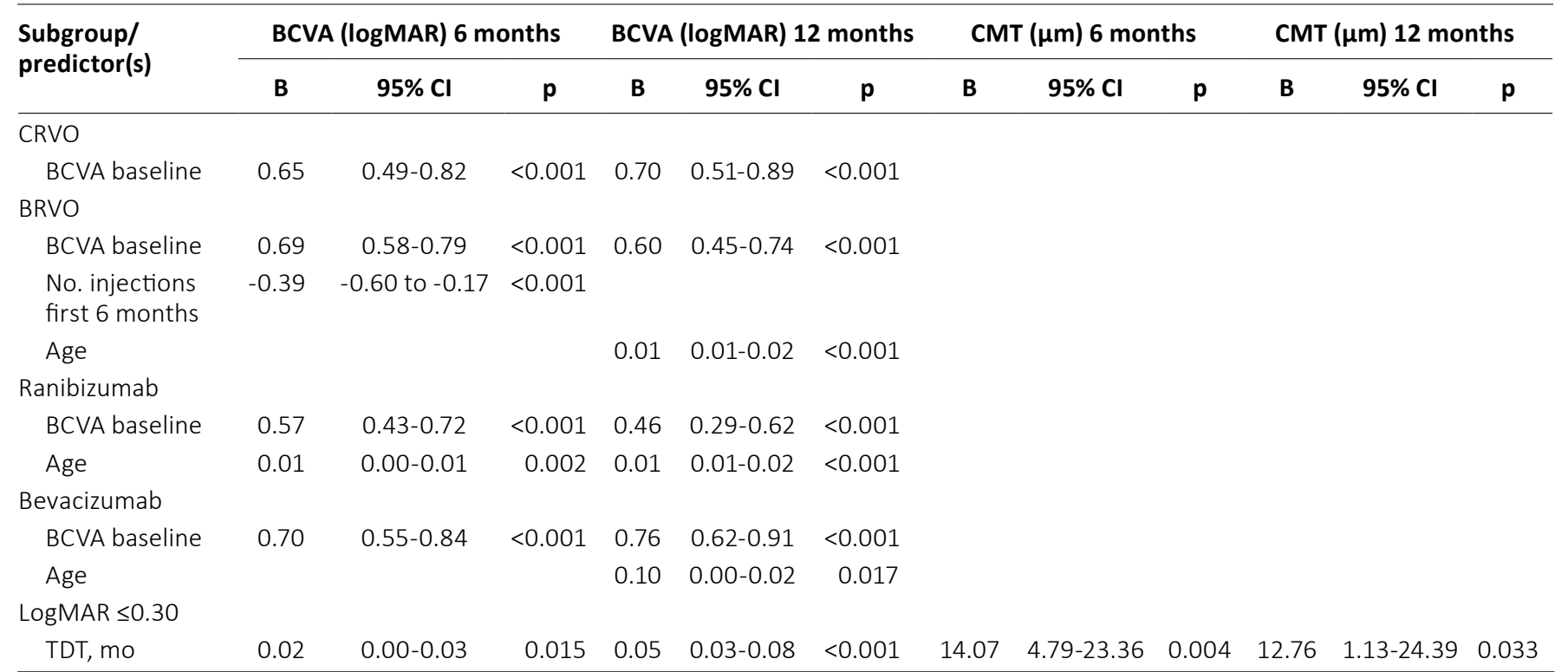

$95 \% \mathrm{Cl}=95 \%$ confidence interval for $\mathrm{B} ; \mathrm{B}=$ unstandardized coefficient; $\mathrm{BRVO}=$ branch retinal vein occlusion; $\mathrm{CRVO}=$ central retinal vein occlusion; logMAR $\leq 0.30=$ good vision at baseline; $\log M A R \geq 1.00$ = poor vision at baseline; TDT = time from diagnosis to treatment.

$86.0)$ vs $70.0(45.0-90.0)$ years $(p=0.016)$. More patients in the poor vision group underwent rescue grid laser photocoagulation during follow-up ( $54.4 \%$ vs $32.4 \%, p=0.040)$.

\section{Number of injections during the 12-month study period}

The median number of injections was the same for the BRVO and the CRVO groups, both during the first 6 months (median of 3 injections) and from 6 to 12 months (median of 1 injection). The median (minimum-maximum) total number of injections for the BRVO group was 4 (1-11) and for the CRVO group 4 (1-12), which was not statistically different. The median (minimum-maximum) number of injections during the first 6 months, from 6 to 12 months, and total number of injections was $3(0-6), 1(0-5)$, and 4 (1-10) in the ranibizumab group and $3(0-7), 1(0-4)$, and $4(1-10)$ in the bevacizumab group ( $p>0.05)$.
No significant differences were seen between the groups with good and poor vision at baseline, with the median (minimummaximum) number of injections during the first 6 months, from 6 to 12 months, and total number of injections being $2.5(0-6), 1(0-5)$, and $3.5(1-11)$ for the good vision group and 3 $(0-7), 1(0-4)$, and 4 (1-10) for the poor vision group.

\section{Regression analyses}

Significant results from the univariate and multivariate regression analyses to determine predictors of BCVA and CMT at 6 and 12 months, depending on 5 of the 6 studied subgroups, are presented in Table V. There were no identified predictors for the group with poor baseline vision. A worse BCVA at baseline was a predictor of a worse BCVA at 6 and 12 months for all groups ( $p<0.001)$, except for the group with 
good vision at baseline. For this group, only a shorter time from diagnosis to treatment was a predictor of better BCVA and CMT, both at 6 and 12 months $(p=0.015$ and $p=0.004$ at 6 months, $p<0.001$ and $p=0.033$ at 12 months). Older age was a predictor of a worse BCVA at 12 months in the BRVO and bevacizumab groups $(p<0.001$ and $p=0.017$, respectively), while in the ranibizumab group it was a predictor of worse BCVA at both 6 and 12 months ( $p=0.002$ and $p<0.001$, respectively). Only in the BRVO group was a lower number of injections during the first 6 months of treatment a predictor of worse BCVA at 6 months $(p<0.001)$.

\section{Discussion}

Our results showed that, regardless of treatment or baseline visual acuity, median BCVA and CMT improved in both the BRVO and CRVO groups at 6 and 12 months compared to baseline. However, while there was no difference in BCVA between 6 and 12 months, the CRVO group continued to show a statistically significant decrease in CMT in this period. Since this CMT reduction was not accompanied by a BCVA change, we may speculate that the structural result better reflected the effect of treatment than the functional result. Brynskov et al (8) reported that, in CRVO, the functional outcome did not mirror the anatomical outcome, and Pielen et al, in the Ranibizumab for Branch Retinal Vein Occlusion Associated Macular Edema Study (RABAMES) (9), showed that, in BRVO, central retinal thickness increased slowly after stopping injections, whereas improvement in visual acuity was sustained, indicating that morphologic changes occur prior to functional impairment (9). In our CRVO population, functional and structural improvement occurred simultaneously until 6 months of follow-up, but after this period only morphologic improvement continued to occur. This may be explained by the duration of the edema and subsequent photoreceptor damage, which could limit complete visual acuity recovery.

When comparing the CRVO group with the BRVO group, median BCVA was better in the BRVO group both at 6 and 12 months, and median CMT was lower at both baseline and 6 months in the same group. Our study population received a median of 3 injections in the first 6 months (bevacizumab or ranibizumab) and 1 injection between 6 and 12 months in both groups.

When comparing bevacizumab vs ranibizumab, our results showed that, although they were both effective in reducing BCVA and CMT in the first 6 months, only ranibizumab further reduced CMT between months 6 and 12. Moreover, CMT was lower in the ranibizumab group when compared to the bevacizumab group, both at 6 and 12 months, with no differences in BCVA between groups. The median number of injections was similar between the ranibizumab and bevacizumab groups, with 3 injections during the first 6 months and 1 injection from 6 to 12 months. Our results are in line with other real-world studies, which have shown that, in patients with RVO, and as early as at 2 weeks postinjection, CMT reduction is higher with ranibizumab compared to bevacizumab (10). Two prospective trials comparing bevacizumab and ranibizumab in the treatment of RVO (11) or BRVO (12) concluded that both agents had similar effects in reducing macular thickness and improving visual acuity at 6 months
(11), even using a PRN regimen, with a mean number of injections of bevacizumab and ranibizumab of 3.2 and 3.0, respectively (12). Therefore, both bevacizumab and ranibizumab are effective, even with real-world treatment regimens using a lower number of injections than recommended.

Our results also showed that more patients in the bevacizumab group underwent rescue grid laser photocoagulation during follow-up (66.3\% vs $29.9 \%, \mathrm{p}<0.001)$. Other studies reported rescue grid laser treatment of 51\% (13) and 42\% (14) for BRVO and $16.5 \%$ (14) for CRVO after bevacizumab therapy, but there are no studies directly comparing bevacizumab with ranibizumab. In our population sample, ranibizumab seemed to prevent the need for rescue laser treatment more efficiently than bevacizumab, but this result has to be confirmed.

One study reported that, in patients with BRVO treated with ranibizumab, patients with worse baseline visual acuity showed greater visual benefit from treatment (15). Our results are in accordance with this study, since only the group with poor vision at baseline had further decreased CMT between 6 and 12 months and improved median BCVA at 6 and 12 months compared to baseline. This may reflect the worse condition of these patients, which, unlike the good vision group, could still improve, as was the case with the CRVO group. These results support our previous suggestion that the structural result better reflects the effect of treatment than the functional result. The group with poor visual acuity at baseline was older and more patients in this group underwent rescue grid laser photocoagulation during follow-up.

We did not identify any predictors of the studied outcomes for the group with poor baseline vision. We may speculate that these patients had such a serious condition that all efforts to improve their status are independent of the predictor variables identified for the other groups. For the other 5 analyzed groups, a worse BCVA at baseline was a predictor of a worse BCVA at 6 and 12 months for all groups, except for the group with good baseline vision, probably because these patients had a smaller margin for improvement. Other studies have reported similar results (16-20). Older age was a predictor of worse BCVA in the BRVO, bevacizumab, and ranibizumab groups, which is in agreement with other reports $(8,16-20)$. Only in the BRVO group was a lower number of injections during the first 6 months of treatment a predictor of worse BCVA at 6 months. This result suggests that these patients are being undertreated and could have improved outcomes with a more intensive approach during the first 6 months of treatment. Several studies have reported early treatment as a predictor of better visual outcomes $(17,21-25)$ for both BRVO and CRVO. Our results show that a shorter time from diagnosis to treatment is only a predictor of better BCVA and CMT, both at 6 and 12 months, in the group with good vision at baseline. This suggests that the therapy should be administered as soon as possible after the diagnosis.

In summary, both treatments were effective, although less effective than results reported in clinical trials. Most patients were treated using a PRN regimen and experienced the constraints of the public health care system and overbooked agendas. It is thus not surprising that real-world patients are undertreated. 


\section{Appendix}

Members of the Portuguese Multicenter Study Group of Retinal Vascular Diseases (PORTUGAL): André Diogo Barata (Department of Ophthalmology, Hospital de Santa Maria, Centro Hospitalar Lisboa Norte, Lisbon; Department of Ophthalmology, Faculty of Medicine, Universidade de Lisboa, Lisbon), Bárbara Borges (Department of Ophthalmology, Centro Hospitalar Lisboa Central, Lisbon), Ana Cabugueira (Department of Ophthalmology, Centro Hospitalar Lisboa Central, Lisbon), Joana Campos (Department of Ophthalmology, Centro Hospitalar de Leiria, Leiria), Rita Matos (Department of Ophthalmology, Centro Hospitalar do Baixo Vouga, Aveiro), Filipe S. Neves (Department of Ophthalmology, Centro Hospitalar Vila Nova de Gaia e Espinho, Vila Nova de Gaia), and Miguel Ruão (Department of Ophthalmology, Centro Hospitalar de Entre o Douro e Vouga, Santa Maria da Feira).

\section{Disclosures}

Financial support: Dr. Vaz-Pereira reports personal fees and nonfinancial support from Bayer and nonfinancial support from Allergan and Novartis, outside the submitted work. Dr. Flores reports personal fees and nonfinancial support from Allergan and Bayer and nonfinancial support from Novartis, outside the submitted work.

Conflict of interest: None of the authors has conflict of interest with this submission.

Meeting presentation: This work was presented as a poster at the $1^{\text {st }}$ scientific meeting of the Portuguese Retina Study Group (GER), Lisbon, February 13, 2016; as a poster at the $16^{\text {th }}$ ESASO Retina Academy, Estoril, Portugal, June 23-25, 2016; and as a presentation at the $16^{\text {th }}$ EURETINA Congress, Copenhagen, September 8-11, 2016.

\section{References}

1. Ho M, Liu DT, Lam DS, Jonas JB. Retinal vein occlusions, from basics to the latest treatment. Retina. 2016;36(3):432-448.

2. Campa C, Alivernini G, Bolletta E, Parodi MB, Perri P. Anti-VEGF TherapyforRetinalVein Occlusions. CurrDrugTargets.2016;17(3): 328-336.

3. European Medicines Agency website. Authorization details of Lucentis $^{\circledR} \quad$ (ranibizumab). www.ema.europa.eu/ema/index.jsp? curl=pages/medicines/human/medicines/000715/human_med 000890.jsp\&mid=WC0b01ac058001d124. Accessed December 14, 2016.

4. European Medicines Agency website. Authorization details of Eylea ${ }^{\circledR}$ (aflibercept). www.ema.europa.eu/ema/index.jsp?curl=pages/ medicines/human/medicines/002392/human_med_001598.jsp\& mid=WCOb01ac058001d124. Accessed December 14, 2016.

5. European Medicines Agency website. Authorization details of Avastin $^{\circledR}$ (bevacizumab). www.ema.europa.eu/ema/index.jsp?curl=pages/medicines/human/medicines/000582/ human med 000663.jsp\&mid=WC0b01ac058001d124. Accessed December 14, 2016.

6. Ashraf M, Souka AA, Singh RP. Central retinal vein occlusion: modifying current treatment protocols. Eye (Lond). 2016; 30(4):505-514.

7. Holladay JT. Visual acuity measurements. J Cataract Refract Surg. 2004;30(2):287-290.

8. Brynskov T, Kemp H, Sørensen TL. Intravitreal ranibizumab for retinal vein occlusion through 1 year in clinical practice. Retina. 2014;34(8):1637-1643.

9. Pielen A, Mirshahi A, Feltgen N, et al; RABAMES Study Group. Ranibizumab for Branch Retinal Vein Occlusion Associated Macular Edema Study (RABAMES): six-month results of a prospective randomized clinical trial. Acta Ophthalmol. 2015;93(1):e29-e37.
10. Singer MA, Cohen SR, Groth SL, Porbandarwalla S. Comparing bevacizumab and ranibizumab for initial reduction of central macular thickness in patients with retinal vein occlusions. Clin Ophthalmol. 2013;7:1377-1383.

11. Rajagopal R, Shah GK, Blinder KJ, et al. Bevacizumab Versus Ranibizumab in the Treatment of Macular Edema Due to Retinal Vein Occlusion: 6-Month Results of the CRAVE Study. Ophthalmic Surg Lasers Imaging Retina. 2015;46(8):844-850.

12. Narayanan R, Panchal B, Das T, Chhablani J, Jalali S, Ali $\mathrm{MH}_{i}$ MARVEL study group. A randomised, double-masked, controlled study of the efficacy and safety of intravitreal bevacizumab versus ranibizumab in the treatment of macular oedema due to branch retinal vein occlusion: MARVEL Report No. 1. Br J Ophthalmol. 2015;99(7):954-959.

13. Siegel RA, Dreznik A, Mimouni K, Bor E, Weinberger D, Bourla DH. Intravitreal bevacizumab treatment for macular edema due to branch retinal vein occlusion in a clinical setting. Curr Eye Res. 2012;37(9):823-829.

14. Kiss S, Liu Y, Brown J, et al. Clinical utilization of anti-vascular endothelial growth-factor agents and patient monitoring in retinal vein occlusion and diabetic macular edema. Clin Ophthalmol. 2014;8:1611-1621.

15. Rouvas A, Petrou P, Ntouraki A, Douvali M, Ladas I, Vergados I. Intravitreal ranibizumab (Lucentis) for branch retinal vein occlusion-induced macular edema: nine-month results of a prospective study. Retina. 2010;30(6):893-902.

16. Ach T, Hoeh AE, Schaal KB, Scheuerle AF, Dithmar S. Predictive factors for changes in macular edema in intravitreal bevacizumab therapy of retinal vein occlusion. Graefes Arch Clin Exp Ophthalmol. 2010;248(2):155-159.

17. Daien V, Navarre S, Fesler P, Vergely L, Villain M, Schneider C. Visual acuity outcome and predictive factors after bevacizumab for central retinal vein occlusion. Eur J Ophthalmol. 2012;22(6):1013-1018.

18. Hoeh AE, Ach T, Schaal KB, Scheuerle AF, Dithmar S. Long-term follow-up of OCT-guided bevacizumab treatment of macular edema due to retinal vein occlusion. Graefes Arch Clin Exp Ophthalmol. 2009;247(12):1635-1641.

19. Jaissle GB, Szurman P, Feltgen N, et al; Retinal Vein Occlusion Study Group. Predictive factors for functional improvement after intravitreal bevacizumab therapy for macular edema due to branch retinal vein occlusion. Graefes Arch Clin Exp Ophthalmol. 2011;249(2):183-192.

20. Kondo $\mathrm{M}$, Kondo $\mathrm{N}$, Ito $\mathrm{Y}$, et al. Intravitreal injection of bevacizumab for macular edema secondary to branch retinal vein occlusion:results after 12 months and multiple regression analysis. Retina. 2009;29(9):1242-1248.

21. Ehlers JP, Decroos FC, Fekrat S. Intravitreal bevacizumab for macular edema secondary to branch retinal vein occlusion. Retina. 2011;31(9):1856-1862.

22. Epstein DL, Algvere PV, von Wendt G, Seregard S, Kvanta A. Benefit from bevacizumab for macular edema in central retinal vein occlusion: twelve-month results of a prospective, randomized study. Ophthalmology. 2012;119(12):2587-2591.

23. Hirose M, Matsumiya W, Honda S, Nakamura M. Efficacy and visual prognostic factors of intravitreal bevacizumab as needed for macular edema secondary to central retinal vein occlusion. Clin Ophthalmol. 2014;8:2301-2305.

24. Thach $A B$, Yau $L$, Hoang $C$, Tuomi L. Time to clinically significant visual acuity gains after ranibizumab treatment for retinal vein occlusion: BRAVO and CRUISE trials. Ophthalmology. 2014;121(5):1059-1066.

25. Yamada R, Nishida A, Shimozono M, et al. Predictive factors for recurrence of macular edema after successful intravitreal bevacizumab therapy in branch retinal vein occlusion. Jpn J Ophthalmol. 2015;59(6):389-393. 